\title{
Effects of kinesiology tape after enucleation of mandibular dentigerous cysts
}

\author{
Min-Gyu Kim, Moon-Young Kim \\ Department of Oral and Maxillofacial Surgery, College of Dentistry, Dankook University, Cheonan, Korea
}

\begin{abstract}
J Korean Assoc Oral Maxillofac Surg 2020;46:108-115)
Objectives: Kinesiology tape (KT) creates a pulling force on the skin, thus improving blood and lymph flow by alleviating hemorrhage and congestion of lymphatic fluid. The authors hypothesized that the use of KT could be beneficial for the management of complications after head and neck surgery and designed this study to evaluate the effects of KT on swelling, pain, and trismus after enucleation of mandibular dentigerous cysts with third molar extraction.
\end{abstract}

Materials and Methods: Forty patients who underwent enucleation of a dentigerous cyst with extraction of the mandibular third molar were selected. The patients were randomized into two groups ( $\mathrm{n}=20$ each): a KT group, where KT was applied after surgery in addition to basic postoperative care, and a control group, where patients received basic postoperative care without KT application. Swelling, pain, and trismus were evaluated before surgery (T0) and on postoperative days 1 (T1), 2 (T2), and 3 (T3). Cyst volume, gauze weight for assessing bleeding, and operation time were recorded.

Results: There was a significant difference between the two groups in the change in swelling up to T1 and the change in swelling between T1 and T2. The maximum swelling in the KT group was significantly less than that in the No-KT group and maximum swelling appeared faster in the KT group than in the No-KT group. Both groups showed a mild pain response but there was no significant difference between the two groups. There was no significant difference on interincisal distance change between the two groups. There were no correlations between cyst volume, bleeding, operation time, and maximum swelling.

Conclusion: KT can effectively manage facial swelling after oral and maxillofacial surgeries such as cyst enucleation and third molar extraction, thus improving postoperative patient satisfaction levels and quality of life.

Key words: Mandible, Odontogenic cysts, Elastic bandage, Postoperative complications

[paper submitted 2019. 8. 6 / revised 2019. 10. 16 / accepted 2019. 10. 17]

\section{Introduction}

Cysts in the oral and maxillofacial region are common ${ }^{1}$. Destruction of surrounding tissues by these cysts can lead to absorption and expansion of the jawbone, pathological fractures, and facial deformations ${ }^{2}$. Cysts can be divided into developmental and inflammatory cysts, and developmental cysts can be further classified into odontogenic and nonodon-

\section{Moon-Young Kim \\ Department of Oral and Maxillofacial Surgery, College of Dentistry, Dankook University, 119 Dandae-ro, Dongnam-gu, Cheonan 31116, Korea TEL: +82-41-550-0271 FAX: +82-41-551-8988 \\ E-mail:kmyomfs@dankook.ac.kr \\ ORCID: https://orcid.org/0000-0001-9596-7481}

(C) This is an open-access article distributed under the terms of the Creative Commons Attribution Non-Commercial License (http://creativecommons.org/ licenses/by-nc/4.0/), which permits unrestricted non-commercial use, distribution, and reproduction in any medium, provided the original work is properly cited.

Copyright (C) 2020 The Korean Association of Oral and Maxillofacial Surgeons. All rights reserved. togenic types. Most developmental cysts are odontogenic and occur more frequently in the jaws than in other parts of the body. A dentigerous cyst is an odontogenic cyst associated with the crown of an unerupted (or partially erupted) tooth. The cyst cavity is lined by epithelial cells derived from the reduced enamel epithelium of the tooth-forming organ. Dentigerous cysts are commonly associated with the mandibular third molars ${ }^{3}$, and most can be completely cured by surgical enucleation, with a relatively low recurrence rate ${ }^{4}$. However, after surgical enucleation, complications such as swelling, pain, and trismus can negatively impact patient quality of life by compromising esthetics, speech, and mastication and causing physical and psychological problems.

During surgeries, inflammatory chemicals such as prostaglandins, leukotrienes, bradykinins, and platelet-activation factors are secreted by damaged tissue. Moreover, excessive lymph production causes disturbances in local lymphatic cir- 
culation. Inflammatory chemicals create endothelial gaps to increase vascular permeability. In addition, macrophages and mast cells in damaged tissues produce histamine, serotonin, and eicosanoids; alter the local constitution of blood vessels; and release nitric oxide, eventually resulting in vasorelaxation. These chain reactions increase blood vessel relaxation and vascular permeability and result in the accumulation of interstitial fluid, eventually resulting in tissue swelling and pain ${ }^{5}$.

Various methods for alleviation of postoperative swelling, pain, and trismus have been studied, including medications such as anti-inflammatory analgesics, steroids, and antibiotics $^{6-9}$; laser treatment ${ }^{10,11}$; and physical therapy such as cryotherapy $^{12}$ or manual lymph drainage ${ }^{13}$. However, no single method can prevent postoperative complications without undesirable side effects or considerable expense. Therefore, superior methods for alleviation of swelling, pain, and trismus after oral and maxillofacial surgery are desirable.

Elastic therapeutic tape, also called kinesiology tape, Kinesio tape, k-tape, or KT, was developed by Dr. K. Kase in the 1970 s with the intent of treating pain and disability associated with sports injuries and other physical disorders. When stretched beyond its normal length and applied, it recoils and creates a pulling force on the skin, thus improving blood and lymph flow by alleviating hemorrhage and congestion of lymphatic fluid ${ }^{14-16}$. Currently, there is limited evidence of its usefulness in the field of oral and maxillofacial surgery.

The authors hypothesized that the use of KT could be beneficial in managing complications after head and neck surgery and designed the present study to evaluate the effects of KT on swelling, pain, and trismus after enucleation of mandibular dentigerous cysts with third molar extraction.

\section{Materials and Methods}

The protocol, survey forms, and consent forms were approved by the Institutional Review Board of Dankook University Dental Hospital (Cheonan, Korea) (No. DKUDH IRB 2019-07-004). This study followed the medical protocol and ethics of the Declaration of Helsinki.

\section{Patients}

In total, 40 patients who underwent enucleation of a dentigerous cyst with extraction of the mandibular third molar in the Department of Oral and Maxillofacial Surgery at Dankook University Dental Hospital during 2018 were selected. The preoperative cyst volume and amount of intraoperative bleeding were quantitatively measured. In addition, swelling, pain, and the mouth opening were assessed for 3 days after surgery. The patients were randomized to two groups ( $\mathrm{n}=20$ each): a KT group, where KT was applied after surgery in addition to basic postoperative care, and a control group, where patients received basic postoperative care without KT application. Patients with hematoma or infection that could affect postoperative swelling were excluded.

\section{Surgical method}

Under general anesthesia with nasal intubation, one oral and maxillofacial surgeon performed enucleation and tooth extraction according to a standardized surgical protocol. The incision extended from the mesial side of the first premolar to a point $1.5 \mathrm{~cm}$ distal to the second molar. Following elevation of the periosteum and bone cutting, enucleation and tooth extraction were performed. The surgical site was irrigated with normal saline, dried, and packed with gauze, which was allowed to absorb blood for 30 seconds and subsequently weighed in order to determine the amount of bleeding. Subsequently, the wound was closed by interrupted 3-0 Vicryl sutures (Ethicon, Somerville, NJ, USA).

\section{Postoperative care}

An ice pack was applied for 6 hours after surgery in all pa-

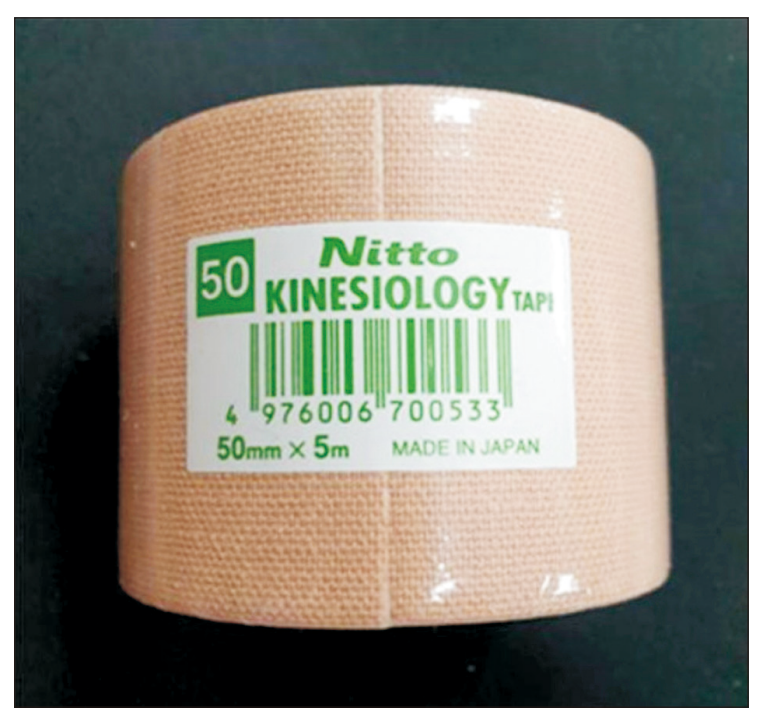

Fig. 1. Skin-colored Nitto Kinesiology Tape $(5 \mathrm{~cm} \times 5 \mathrm{~m}$; Nitto Denko Group).

Min-Gyu Kim et al: Effects of kinesiology tape after enucleation of mandibular dentigerous cysts. JKorean Assoc Oral Maxillofac Surg 2020 
tients, with alternating periods of application for 30 minutes and rest for 30 minutes. For analgesia, all patients received intravenous tramadol hydrochloride $(50 \mathrm{mg} / \mathrm{mL}$, Tridol injection $50 \mathrm{mg}$; Yuhan, Seoul, Korea) 3 times a day for 3 days. Antibiotic treatment involved intravenous ceftriaxone sodium hydrate (1 g, Ceftriaxone injection $1 \mathrm{~g}$; Dongkook, Seoul, Korea) 2 times a day for 3 days. All patients were instructed to gargle with $0.12 \%$ chlorhexidine mouthwash daily in order to maintain cleanliness at the surgical site.

\section{Taping}

All patients in the KT group received skin-colored Nitto Kinesiology Tape ( $5 \mathrm{~cm} \times 5 \mathrm{~m}$; Nitto Denko Group, Osaka, Japan) (Fig. 1) that was applied by a single investigator. The tape was applied in three lines from the clavicle to the traguscommissure line. The base was placed slightly above the area drained by the supraclavicular nodes. Overall, tape placement was directed at the appropriate lymphatic ducts crossing the cervical, submental, submandibular, and parotid nodes.(Fig. 2) Every corner of the tape was rounded while cutting in order to prevent premature peeling of the edges.

\section{Data collection}

Swelling, pain, and trismus were evaluated by a single investigator before surgery (T0) and on postoperative days 1

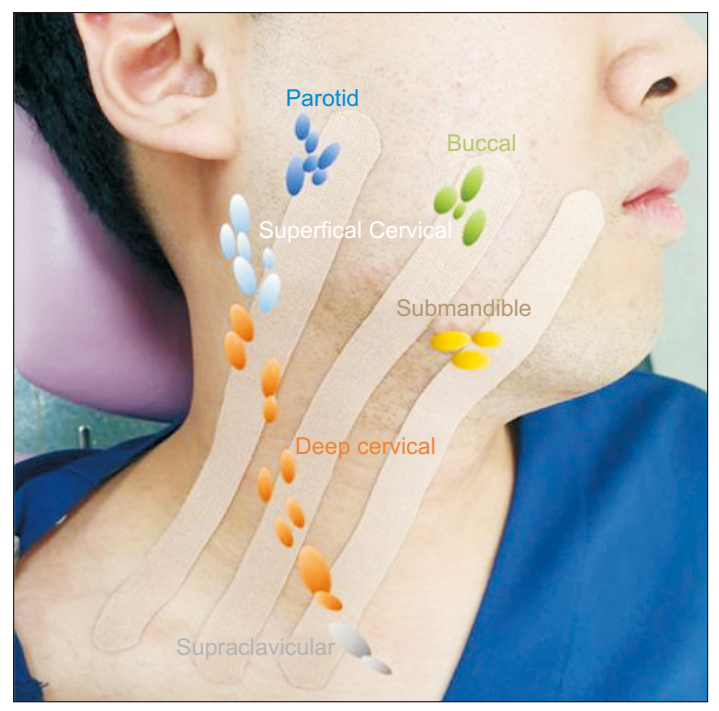

Fig. 2. Method of taping and location of facial lymph nodes. Application of Kinesio tape from above the clavicle to tragus-commissure line. Tape strips stimulate the drainage of swelling and hematoma through lymphatic duct.

Min-Gyu Kim et al: Effects of kinesiology tape after enucleation of mandibular dentigerous cysts. J Korean Assoc Oral Maxillofac Surg 2020
(T1), 2 (T2), and 3 (T3).

\section{1) Swelling}

Swelling was assessed by the four-line measurement method using a standard plastic tape placed in contact with the skin. The measurement lines were as follows: line A, most posterior point of the tragus to the most lateral point of the lip commissure; line B, most posterior point of the tragus to the pogonion; line $\mathrm{C}$, lateral canthus of the eye to the most inferior point of the mandibular angle; and line $\mathrm{D}$, most inferior point of the mandibular angle to the midpoint of the nasal bone.(Fig. 3) The sum of the measured values $(\mathrm{cm})$ for the four lines was calculated at T0, T1, T2, and T3.

\section{2) Pain}

Pain was subjectively assessed using a visual analogue scale (VAS) from 0 to 10 , with patients asked to mark the point corresponding to the intensity of their pain.

\section{3) Trismus}

The maximum mouth opening was recorded by measurement of the maximum interincisal distance ( $\mathrm{mm}$ ) using calipers.

\section{4) Cyst volume}

Preoperative cone-beam computed tomography images were obtained using the Alphard VEGA system (Asahi, Tokyo, Japan). Invivo 5.1 software (Anatomage, San Jose, CA,

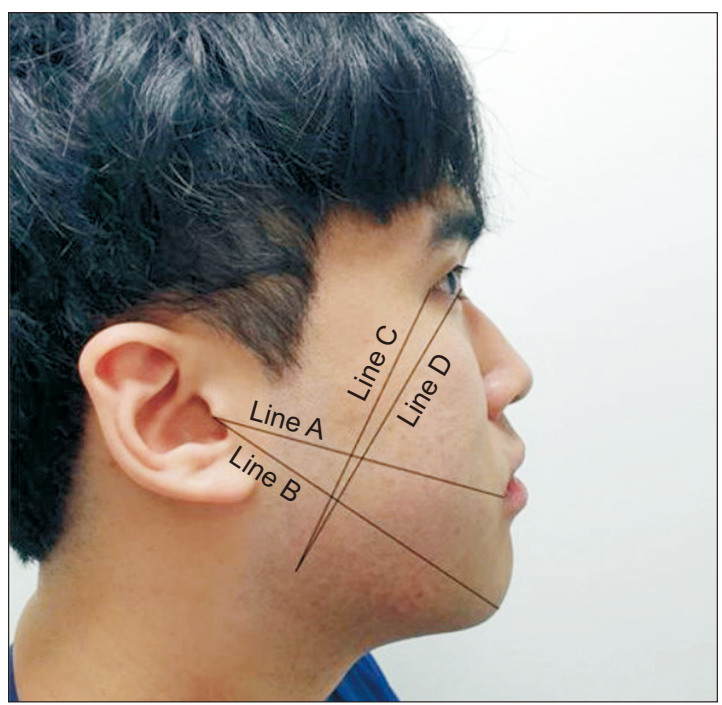

Fig. 3. The four-line measurement. Segment endpoints were marked to ensure accurate measurements.

Min-Gyu Kim et al: Effects of kinesiology tape after enucleation of mandibular dentigerous cysts. J Korean Assoc Oral Maxillofac Surg 2020 
USA) was used for cyst volume measurement $\left(\mathrm{mm}^{3}\right)$ based on the radiolucent border.

\section{5) Intraoperative bleeding}

After cyst enucleation and tooth extraction, the surgical site was cleaned with normal saline and dried using gauze. Subsequently, gauze was packed into the surgical site for 30 seconds, and the amount of bleeding was measured through the weight of the gauze $(\mathrm{g})$.

\section{Data analysis}

Two-sided independent sample t-tests were used to determine differences between the KT and control groups. Repeated measures ANOVA was used to observe changes in the degree of swelling between the two groups, while Pearson's correlation analysis was used to analyze the relationship between the maximum swelling and the preoperative cyst volume, and amount of intraoperative bleeding. All statistical analyses were performed using IBM SPSS Statistics (ver.
20.0; IBM, Armonk, NY, USA). The significance level was set at $5 \%$.

\section{Results}

\section{Baseline characteristics}

The study included 29 males (72.5\%) and 11 females $(27.5 \%)$ with a mean age of $39.2 \pm 14.36$ years. The clinical and demographic characteristics of the 40 patients are listed in Table 1. The KT and control groups were similar in terms

Table 4. Visual analogue scale (VAS) scores

\begin{tabular}{cccc}
\hline VAS score & KT group & Control group & $P$-value \\
\hline T1 & $1.5 \pm 1.9$ & $2.5 \pm 1.7$ & $>0.05$ \\
T2 & $2.2 \pm 1.7$ & $3.5 \pm 2.7$ & $>0.05$ \\
T3 & $2.1 \pm 1.6$ & $2.0 \pm 1.5$ & $>0.05$ \\
\hline
\end{tabular}

(KT: kinesiology tape)

Values are presented as mean \pm standard deviation.

Min-Gyu Kim et al: Effects of kinesiology tape after enucleation of mandibular dentigerous cysts. J Korean Assoc Oral Maxillofac Surg 2020

Table 1. Demographics and baseline characteristics

\begin{tabular}{|c|c|c|c|}
\hline & KT group $(n=20)$ & Control group $(n=20)$ & $P$-value \\
\hline Age (yr) & $42.35 \pm 103.39$ & $36.05 \pm 289.1$ & $>0.05$ \\
\hline Male $(\%)$ & $15(75.0)$ & $14(70.0)$ & $>0.05$ \\
\hline Swelling at T0 (cm) & $54.55 \pm 2.24$ & $54.36 \pm 3.51$ & $>0.05$ \\
\hline IID at T0 $(\mathrm{mm})$ & $49.11 \pm 21.77$ & $49.60 \pm 25.81$ & $>0.05$ \\
\hline Cyst volume $\left(\mathrm{mm}^{3}\right)$ & $7.56 \pm 67.31$ & $11.63 \pm 345.43$ & $>0.05$ \\
\hline Amount of bleeding (g) & $0.58 \pm 0.17$ & $0.48 \pm 0.05$ & $>0.05$ \\
\hline Operation duration (min) & $46.65 \pm 129.18$ & $44.25 \pm 161.77$ & $>0.05$ \\
\hline
\end{tabular}

(KT: kinesiology tape, IID: interincisal distance)

Values are presented as mean \pm standard deviation or number (\%).

Min-Gyu Kim et al: Effects of kinesiology tape affer enucleation of mandibular dentigerous cysts. J Korean Assoc Oral Maxillofac Surg 2020

Table 2. Swelling change from before the procedure to one day after

\begin{tabular}{cccc}
\hline Swelling change & KT group & Control group & $P$-value \\
\hline T1 to T0 $(\mathrm{cm})$ & $2.65 \pm 1.93$ & $3.66 \pm 1.06$ & $<0.05^{*}$ \\
T2 to T1 $(\mathrm{cm})$ & $0.42 \pm 1.28$ & $2.06 \pm 0.91$ & $<0.05^{*}$ \\
T3 to T2 $(\mathrm{cm})$ & $-0.77 \pm 1.35$ & $-0.56 \pm 0.89$ & $>0.05$ \\
\hline
\end{tabular}

(KT: kinesiology tape)

*Significant at the level of $P<0.05$.

Values are presented as mean \pm standard deviation.

Min-Gyu Kim et al: Effects of kinesiology tape after enucleation of mandibular dentigerous cysts. J Korean Assoc Oral Maxillofac Surg 2020

Table 3. Maximum swelling

\begin{tabular}{llr}
\hline & KT group & Control group \\
\hline Maximum swelling $(\mathrm{cm})$ & $3.46 \pm 4.06$ & $5.92 \pm 2.98$ \\
Time to maximum swelling (day) & $1.75 \pm 0.61$ & $2.25 \pm 0.19$ \\
\hline
\end{tabular}

(KT: kinesiology tape)

*Significant at the level of $P<0.05$.

Values are presented as mean \pm standard deviation.

Min-Gyu Kim et al: Effects of kinesiology tape affer enucleation of mandibular dentigerous cysts. J Korean Assoc Oral Maxillofac Surg 2020 
of sex distribution, age, the amount of swelling at T0, and interincisal distance at T0, cyst volume, and the amount of bleeding. The average hospital stay was 3.77 days (minimum 3 days, maximum 7 days).

\section{Swelling}

The change in the degree of swelling from T0 to T1 and from $\mathrm{T} 1$ to $\mathrm{T} 2$ was significantly different between the KT and control groups, while there was no significant intergroup difference in the change from T2 to T3.(Table 2)

The maximum swelling measured $3.46 \pm 4.06 \mathrm{~cm}$ and $5.92 \pm 2.98 \mathrm{~cm}$ in the KT and control groups, respectively, with a statistically significant difference between groups. Moreover, maximum swelling appeared significantly earlier in the KT group ( $1.75 \pm 0.61$ days) than in the control group (2.25 \pm 0.19 days).(Table 3$)$

\section{Pain}

Both groups showed a mild pain response after surgery, with no statistically significant differences in VAS score at

Table 5. Change of interincisal distance (IID) from before the procedure to one day after

\begin{tabular}{cccc}
\hline IID change & KT group & Control group & $P$-value \\
\hline T1 to T2 $(\mathrm{mm})$ & $0.10 \pm 16.51$ & $1.40 \pm 10.25$ & $>0.05$ \\
T2 to T3 $(\mathrm{mm})$ & $2.01 \pm 6.21$ & $1.25 \pm 1.35$ & $>0.05$ \\
\hline
\end{tabular}

(KT: kinesiology tape)

Values are presented as mean \pm standard deviation.

Min-Gyu Kim et al: Effects of kinesiology tape after enucleation of mandibular dentigerous cysts. J Korean Assoc Oral Maxillofac Surg 2020 any time point.(Table 4$)$

\section{Trismus}

The maximum mouth opening showed no significant intergroup difference at any time point.(Table 5)

\section{Cyst volume}

Pearson's correlation analysis showed no significant correlation between preoperative cyst volume and the maximum swelling after surgery in either group.(Table 6)

\section{Bleeding}

Pearson's correlation analysis showed no significant correlation between the amount of intraoperative bleeding and the maximum swelling after surgery in either group.(Table 7)

\section{Discussion}

In the present study, the authors found that the use of KT (KT group) after enucleation of a dentigerous cyst with mandibular third molar extraction resulted in superior outcomes in terms of the change in the degree of swelling and maximum swelling after surgery. However, pain and trismus did not vary between patients who received the tape and those who did not. Moreover, there was no correlation between the maximum swelling after surgery and preoperative cyst volume or amount of intraoperative bleeding.

Table 6. Pearson correlation analysis between cyst volume and maximum swelling

\begin{tabular}{llcc}
\hline & & Maximum swelling & Cyst volume \\
\hline Maximum swelling & Pearson correlation coefficient & 1 & 0.162 \\
& $P$-value & - & 0.317 \\
Cyst volume & Number & 40 & 40 \\
& Pearson correlation coefficient & 0.162 & 1 \\
& $P$-value & 0.317 & - \\
& Number & 40 & 40 \\
\hline
\end{tabular}

Min-Gyu Kim et al: Effects of kinesiology tape after enucleation of mandibular dentigerous cysts. J Korean Assoc Oral Maxillofac Surg 2020

Table 7. Pearson correlation analysis between amount of bleeding and maximum swelling

\begin{tabular}{clcc}
\hline & & Maximum swelling & Amount of bleeding \\
\hline Maximum swelling & Pearson correlation coefficient & 0.007 & - \\
& $P$-value & 0.964 & 40 \\
Amount of bleeding & Number & 40 & 0.007 \\
& Pearson correlation coefficient & 0.964 & 40 \\
\hline
\end{tabular}

Min-Gyu Kim et al: Effects of kinesiology tape after enucleation of mandibular dentigerous cysts. J Korean Assoc Oral Maxillofac Surg 2020 
Numerous studies have evaluated measures for the management of complications after oral and maxillofacial surgeries. The use of laser therapy after third molar extraction is painless and noninvasive, with no reported adverse effects. Laser therapy increases the number and diameter of lymph vessels and simultaneously decreases vascular permeability. However, this treatment requires special equipment and is expensive $^{10}$. Cryotherapy is a simple, cheap, and repeatable tool that works by changing the hematic flow, causing vasoconstriction and lowering the metabolic rate, and thus reducing bacterial growth. However, the effects of cryotherapy remain controversial $^{12}$. Several researchers have advocated the use of corticosteroids to limit postoperative complications. Corticosteroids prevent the production of inflammatory factors, including cytokines. Brucoli et al. ${ }^{17}$ claimed that steroids were effective for alleviating pain, swelling, and other symptoms after oral and maxillofacial surgery. However, the side effects of long-term corticosteroid use, such as immunosuppression, adrenal insufficiency, obesity, and diabetes, cannot be ignored. Furthermore, there are limited randomized clinical trials showing evidence of their effectiveness. Szolnoky et al. ${ }^{13}$ claimed that lymphatic massage promotes lymphatic circulation and consequently reduces facial swelling and pain after third molar extraction. However, lymphatic massage can only be performed by a professional therapist. Moreover, it is expensive and requires direct physical manipulation.

Developed by Dr. K. Kase in the 1970s, KT techniques have been shown to support injured muscles and joints ${ }^{16}$. Several studies have attempted to demonstrate the effectiveness of KT in the medical field. Nelson ${ }^{18}$ found that appropriate application of KT can reduce lower limb edema and lumbosacral pain. In addition, studies have evaluated the use of KT for reducing lymphatic edema after mastectomy ${ }^{19}$. $\mathrm{Choi}^{20}$ proposed that the application of KT could reduce premenstrual pain, while Chen et al. ${ }^{21}$ and Added et al. ${ }^{22}$ showed that the application of KT could reduce pain in the cervical spine. However, there have been few studies on the usefulness of $\mathrm{KT}$ in the field of oral and maxillofacial surgery. Accordingly, the findings of the present study are expected to make a novel contribution to the literature.

The accurate measurement of postoperative swelling has been a major obstacle in past studies in terms of its objectiveness. Although numerous methods have been tried, most are imprecise, complex, expensive, or difficult to standardize $\mathrm{e}^{23-25}$. In the present study, we attempted to quantify changes in the degree of swelling by using a modified linear measurement technique based on defined landmarks covering the entire area of interest on the face. Extra care was taken to measure the same distance at the time of marking the endpoint of the segment. Furthermore, a single investigator performed all swelling measurements in order to prevent variation.

The results of the present study may be attributed to the unique elastic properties of KT that allow it to structurally lift the skin and open the superficial lymphatic pathways in the affected area. It is believed that KT increases the interstitial space between the skin and underlying connective tissue, thus promoting the flow of blood and lymphatic fluid. Shim et al. ${ }^{26}$ performed a pilot animal study and found that KT affected lymph formation by opening the microvalves in the initial lymphatic vessels, thereby generating appropriate dynamic pressure variation and causing adequate periodic compression and decompression of the superficial and deep initial lymphatics.

Kase et al. ${ }^{16}$ claimed that KT can decrease pain by reducing pressure on nociceptors. However, similar to other investigations ${ }^{27,28}$, the present study showed no significant difference in the degree of pain control between patients who received $\mathrm{KT}$ and those who did not. This could be attributed to the administration of standardized analgesic treatment after surgery and during hospitalization for all patients. Further studies should eliminate standardized analgesic treatment where possible and provide only rescue drugs.

We anticipated that mouth opening after surgery would normalize faster in the KT group than in the control group in the present study. However, there was no significant difference between the two groups.

We found no correlation between maximum swelling after surgery and preoperative cyst volume. Swelling can be defined as an inflammatory, edematous response to surgical trauma ${ }^{29}$. Drew ${ }^{5}$ mentioned that the incision design and method of suturing the oral mucosa can influence postoperative swelling, pain, and trismus. Borgonovo et al ${ }^{30}$ studied differences in comfort levels after third molar extraction using three different incision patterns and found the extent of the incision, periosteal detachment, and masticatory muscle damage to vary according to the incision pattern; all of these factors can affect postoperative swelling, pain, and trismus.

Finally, no correlation was found between maximum swelling and the amount of intraoperative bleeding, most likely because the amount of bleeding and surgical duration did not vary significantly between groups. 


\section{Conclusion}

In conclusion, findings of the present study suggest that $\mathrm{KT}$, which is easy to apply in the head and neck region, and is noninvasive, economical, and complication-free, can effectively manage facial swelling after oral and maxillofacial surgeries such as cyst enucleation and third molar extraction, thus improving postoperative patient satisfaction levels and quality of life.

\section{ORCID}

Min-Gyu Kim, https://orcid.org/0000-0003-2110-6819

Moon-Young Kim, https://orcid.org/0000-0001-9596-7481

\section{Authors' Contributions}

M.G.K. participated in data collection and wrote the manuscript and performed statistical analysis. M.Y.K. participated in the study design and helped to draft the manuscript. All authors read and approved the final manuscript.

\section{Acknowledgements}

This work was supported by a National Research Foundation of Korea (NRF) grant funded by the Korean government (MSIT) (No. NRF-2017R1C1B5076179).

\section{Ethics Approval and Consent to Participate}

The protocol, survey forms, and consent forms were approved by the Institutional Review Board of Dankook University Dental Hospital (Cheonan, Korea) (No. DKUDH IRB 2019-07-004), and the informed consent was waived.

\section{Consent for Publishing Photographs}

Written informed consent was obtained from the patient for publication of this article and accompanying images.

\section{Conflict of Interest}

No potential conflict of interest relevant to this article was reported.

\section{References}

1. Weir JC, Davenport WD, Skinner RL. A diagnostic and epidemiologic survey of 15,783 oral lesions. J Am Dent Assoc 1987:115:439-42.

2. Al Sheddi MA. Odontogenic cysts. A clinicopathological study. Saudi Med J 2012;33:304-8.

3. Ochsenius G, Escobar E, Godoy L, Peñafiel C. Odontogenic cysts: analysis of 2,944 cases in Chile. Med Oral Patol Oral Cir Bucal 2007;12:85-91.

4. Bilodeau EA, Collins BM. Odontogenic cysts and neoplasms. Surg Pathol Clin 2017;10:177-222.

5. Drew SJ. Best practices for management of pain, swelling, nausea, and vomiting in dentoalveolar surgery. Oral Maxillofac Surg Clin North Am 2015;27:393-404.

6. Kim K, Brar P, Jakubowski J, Kaltman S, Lopez E. The use of corticosteroids and nonsteroidal antiinflammatory medication for the management of pain and inflammation after third molar surgery: a review of the literature. Oral Surg Oral Med Oral Pathol Oral Radiol Endod 2009; 107:630-40.

7. Aznar-Arasa L, Harutunian K, Figueiredo R, Valmaseda-Castellón E, Gay-Escoda C. Effect of preoperative ibuprofen on pain and swelling after lower third molar removal: a randomized controlled trial. Int J Oral Maxillofac Surg 2012;41:1005-9.

8. Markiewicz MR, Brady MF, Ding EL, Dodson TB. Corticosteroids reduce postoperative morbidity after third molar surgery: a systematic review and meta-analysis. J Oral Maxillofac Surg 2008;66:1881-94

9. Kyzas PA. Use of antibiotics in the treatment of mandible fractures: a systematic review. J Oral Maxillofac Surg 2011;69:1129-45.

10. Markovic A, Todorovic Lj. Effectiveness of dexamethasone and low-power laser in minimizing oedema after third molar surgery: a clinical trial. Int J Oral Maxillofac Surg 2007;36:226-9.

11. Røynesdal AK, Björnland T, Barkvoll P, Haanaes HR. The effect of soft-laser application on postoperative pain and swelling. A doubleblind, crossover study. Int J Oral Maxillofac Surg 1993;22:242-5.

12. Rana M, Gellrich NC, von See C, Weiskopf C, Gerressen M, Ghassemi A, et al. 3D evaluation of postoperative swelling in treatment of bilateral mandibular fractures using 2 different cooling therapy methods: a randomized observer blind prospective study. J Craniomaxillofac Surg 2013;41:e17-23.

13. Szolnoky G, Szendi-Horváth K, Seres L, Boda K, Kemény L. Manual lymph drainage efficiently reduces postoperative facial swelling and discomfort after removal of impacted third molars. Lymphology 2007;40:138-42.

14. Ulu M, Gözlüklü Ö, Kaya Ç, Ünal N, Akçay H. Three-dimensional evaluation of the effects of Kinesio taping on postoperative swelling and pain after surgically assisted rapid palatal expansion. J Oral Maxillofac Res 2018;9:e3.

15. Ristow O, Pautke C, Kehl V, Koerdt S, Hahnefeld L, HohlwegMajert B. Kinesiologic taping reduces morbidity after oral and maxillofacial surgery: a pooled analysis. Physiother Theory Pract 2014;30:390-8.

16. Kase K, Wallis J, Kase T. Clinical therapeutic applications of the Kinesio ${ }^{\circledR}$ Taping method. Tokyo, Japan: Kinesio Taping Association; 2003.

17. Brucoli M, De Andreis M, Bonaso M, Boffano P, Benech A. Comparative assessment of dexamethasone administration routes for the management of postoperative symptoms following third molar surgery. J Stomatol Oral Maxillofac Surg 2019;120:529-33.

18. Nelson NL. Kinesio taping for chronic low back pain: a systematic review. J Bodyw Mov Ther 2016;20:672-81.

19. Tantawy SA, Kamel DM. The effect of kinesio taping with exercise compared with exercise alone on pain, range of motion, and disability of the shoulder in postmastectomy females: a randomized control trial. J Phys Ther Sci 2016;28:3300-5. 
20. Choi JH. Effects of kinesio taping and hot packs on premenstrual syndrome in females. J Phys Ther Sci 2017;29:1514-7.

21. Chen SM, Alexander R, Lo SK, Cook J. Effects of Functional Fascial Taping on pain and function in patients with non-specific low back pain: a pilot randomized controlled trial. Clin Rehabil 2012;26:924-33.

22. Added MA, Costa LO, Fukuda TY, de Freitas DG, Salomão EC, Monteiro RL, et al. Efficacy of adding the Kinesio Taping method to guideline-endorsed conventional physiotherapy in patients with chronic nonspecific low back pain: a randomised controlled trial. BMC Musculoskelet Disord 2013;14:301.

23. Bjorn H, Lundqvist C, Hjelmstrom P. A photogrammetric method of measuring the volume of facial swellings. J Dent Res 1954;33:295-308.

24. Milles M, Desjardins PJ, Pawel HE. The facial plethysmograph: a new instrument to measure facial swelling volumetrically. J Oral Maxillofac Surg 1985;43:346-52.

25. Van Gool AV, Ten Bosch JJ, Boering G. A photographic method of assessing swelling following third molar removal. Int J Oral Surg 1975;4:121-9.

26. Shim JY, Lee HR, Lee DC. The use of elastic adhesive tape to promote lymphatic flow in the rabbit hind leg. Yonsei Med J 2003;44:1045-52.

27. González-Iglesias J, Fernández-de-Las-Peñas C, Cleland JA,
Huijbregts P, Del Rosario Gutiérrez-Vega M. Short-term effects of cervical kinesio taping on pain and cervical range of motion in patients with acute whiplash injury: a randomized clinical trial. J Orthop Sports Phys Ther 2009;39:515-21.

28. Thelen MD, Dauber JA, Stoneman PD. The clinical efficacy of kinesio tape for shoulder pain: a randomized, double-blinded, clinical trial. J Orthop Sports Phys Ther 2008;38:389-95.

29. Barbalho JC, Vasconcellos RJ, de Morais HH, Santos LA, Almeida RA, Rêbelo HL, et al. Effects of co-administered dexamethasone and nimesulide on pain, swelling, and trismus following third molar surgery: a randomized, triple-blind, controlled clinical trial. Int J Oral Maxillofac Surg 2017;46:236-42.

30. Borgonovo AE, Giussani A, Grossi GB, Maiorana C. Evaluation of postoperative discomfort after impacted mandibular third molar surgery using three different types of flap. Quintessence Int 2014;45:319-30.

How to cite this article: Kim MG, Kim MY. Effects of kinesiology tape after enucleation of mandibular dentigerous cysts. J Korean Assoc Oral Maxillofac Surg 2020;46:108-115. https://doi. org/10.5125/jkaoms.2020.46.2.108 